\title{
Older adults demonstrate interlimb transfer of reactive gait adaptations to repeated unpredictable gait perturbations
}

\author{
Christopher McCrum (D) Kiros Karamanidis • \\ Lotte Grevendonk • Wiebren Zijlstra • \\ Kenneth Meijer (i)
}

Received: 8 October 2019 / Accepted: 22 October 2019 / Published online: 27 November 2019

(C) The Author(s) 2019

\begin{abstract}
The ability to rapidly adjust gait to cope with unexpected mechanical perturbations declines with ageing. Previous studies, however, have not ensured that gait stability pre-perturbation was equivalent across participants or age groups which may have influenced the outcomes. In this study, we investigate if age-related differences in stability following gait perturbations remain when all participants walk with equivalent stability. We also examine if interlimb transfer of gait adaptations are observed in healthy older adults, by examining if adaptation to repeated perturbations of one leg can benefit stability recovery when the other leg is perturbed. During walking at their stability-normalised walking speeds (young: $1.32 \pm 0.07 \mathrm{~m} / \mathrm{s}$; older: $1.31 \pm$ $0.13 \mathrm{~m} / \mathrm{s}$; normalised to an average margin of stability of $0.05 \mathrm{~m}$ ), 30 young and 28 older healthy adults experi-
\end{abstract}

Electronic supplementary material The online version of this article (https://doi.org/10.1007/s11357-019-00130-x) contains supplementary material, which is available to authorized users.

C. McCrum $(\bowtie) \cdot$ L. Grevendonk $\cdot$ K. Meijer

Department of Nutrition and Movement Sciences, NUTRIM

School of Nutrition and Translational Research in Metabolism,

Maastricht University Medical Centre+, PO Box 616, 6200

MDMaastricht, The Netherlands

e-mail: chris.mccrum@maastrichtuniversity.nl

C. McCrum • W. Zijlstra

Institute of Movement and Sport Gerontology, German Sport

University Cologne, Cologne, Germany

K. Karamanidis

Sport and Exercise Science Research Centre, School of Applied

Sciences, London South Bank University, London, UK enced ten unpredictable treadmill belt accelerations (the first and last applied to the right leg, the others to the left leg). Using kinematic data, we assessed the margins of stability during unperturbed walking and the first eight post-perturbation recovery steps. Older adults required three more steps to recover during the first perturbation to each leg than the young adults. Yet, after repeated perturbations of the left leg, older adults required only one more step to recover. Interestingly, for the untrained right leg, the older adults could regain stability with three fewer steps, indicating interlimb transfer of the improvements. Age differences in reactive gait stability remain even when participants' walk with equivalent stability. Furthermore, we show that healthy older adults can transfer improvements in balance recovery made during repeated perturbations to one limb to their recovery following a perturbation to the untrained limb.

Keywords Aged B Balance · Falls · Locomotion ·

Stability

\section{Introduction}

The ability to maintain or regain gait stability following unexpected mechanical perturbations such as trips, slips or ground surface changes is negatively affected in older age (Bierbaum et al. 2010; Pavol et al. 2002; Süptitz et al. 2013) which reflects older adults' increased risk of falling during walking (Berg et al. 1997; Talbot et al. 2005). However, ageing does not greatly affect the ability to improve balance recovery responses to 
unexpected perturbations with repeated practice, nor the retention of these improvements over time (Bhatt et al. 2012; Epro et al. 2018a, b; Pai et al. 2014). This has led to the development of perturbation-based balance training interventions, whereby different perturbations can be experienced and used as a training stimulus in a safe, controlled environment (Gerards et al. 2017).

There is reasonable evidence in humans that increasing motor error during locomotion (i.e. mismatch between expected sensory feedback based on the feedforward predictions of a normal gait pattern and the actual sensory feedback obtained following a perturbation to normal gait) facilitates greater or faster adaptation (Emken and Reinkensmeyer 2005; Kao et al. 2013). Motor error during a gait-like task in a stepping robot improves motor learning in young adults (Marchal-Crespo et al. 2017; Marchal-Crespo et al. 2014). Furthermore, adaptation to split-belt and forcefield perturbations during walking, as well as subsequent retention and savings (faster adaptation on reexposure to a perturbation) of these adaptations, often occur to a greater extent following abrupt versus gradual exposure to the perturbations (Roemmich and Bastian 2015; Torres-Oviedo and Bastian 2012; van Asseldonk et al. 2011). Additionally, experiencing large, rather than small, perturbations in an initial task leads to better generalisation to other similar locomotor tasks in both split-belt walking (Leech et al. 2018) and slip-perturbed walking (Liu et al. 2016). These results indicate that older adults who experience more balance loss or difficulty recovering from gait perturbations may experience larger motor errors (more diversion from the intended regular gait pattern) and experience a relatively larger stimulus for adaptation than younger adults completing the same gait perturbation task. In previous gait perturbation studies, transfer between similar perturbation tasks has been observed (Bieryla et al. 2007; Parijat and Lockhart 2012; Yang et al. 2013), but there is only limited evidence of interlimb transfer of reactive gait adaptations to perturbations (Bhatt and Pai 2008; McCrum et al. 2018). However, in both previous interlimb studies, only young healthy participants were included. If older adults had been exposed to similar perturbation conditions, the extent of motor errors experienced may have been much greater.

Walking speed can influence the impact of, and the response to, different perturbations (Bhatt et al. 2005; Espy et al. 2010; Pavol et al. 1999). If the same speed is used for all participants, this may result in different degrees of task difficulty (McCrum et al. 2017; McCrum et al. 2019b). In this study, we first aimed to determine if age-related differences in reactive gait stability and its adaptability in response to repeated mechanical gait perturbations are found when the participants' walking speed is normalised to gait stability. To achieve this, we applied our recently published method of walking speed normalisation which reduces interparticipant differences in gait stability (McCrum et al. 2019b) assessed by the margins of stability (MoS; Hof et al. 2005). With this method, multiple trials at different walking speeds are conducted, from which a speed that results in an equivalent baseline level of gait stability across participants is calculated using a polynomial function. As a result, we can infer that any differences in the response to the perturbations are not, in whole or part, artefacts of the walking speeds of the participants, but rather due to true differences in recovery responses. Based on previous work in trips leading to forward balance loss (Epro et al. 2018a; Süptitz et al. 2013), we hypothesised that older adults would require more steps to regain stability than younger adults following the initial perturbation to each leg, despite the stabilitynormalised walking speed, but that both groups would be able to adapt their gait to improve stability during the repetitions to the left leg. The second aim was to determine if interlimb transfer of these adaptations could be observed in healthy older adults, despite the limited support in young adults in our previous study (McCrum et al. 2018). Given our expectation that older adults would require more steps to regain stability than younger adults following the initial perturbation to each leg, and thereby experience greater motor error in their early responses, we hypothesised that evidence of interlimb transfer would be found in the older adults.

\section{Methods}

\section{Participants}

Thirty healthy young adults (12 males, 18 females; age, $24 \pm 2.5$ years; height, $173 \pm 8 \mathrm{~cm}$; weight, $71 \pm 13.9 \mathrm{~kg}$ ) and twenty-eight healthy older adults (17 males, 11 females; age: $71 \pm 4$ years; height: $169 \pm 9.3 \mathrm{~cm}$; weight: $76 \pm 11.9 \mathrm{~kg}$ ) participated in this study. Participants were recruited via posters placed around the university and in local gyms and fitness centres. Data from 18 healthy young adults have been reported in our previous study 
(McCrum et al. 2018) as part of a different analysis. While this was a convenience sample taken from a larger study powered for a different outcome, 28 to 30 participants provide sufficient power $(0.72<\beta<0.96)$ to detect the moderate to large effect sizes of interest (Cohen's $d$ of 0.5-0.7) that we observed in our previous study (McCrum et al. 2018). The participants had no self-reported history of walking difficulties, dizziness or balance problems, had no known neuromuscular condition or injury that could affect balance or walking and could walk at a regular pace for 30 minutes without assistance and without stopping. Written informed consent was obtained, and the study was conducted in accordance with the Declaration of Helsinki. The study protocol was approved by the Maastricht University Medical Centre medical ethics committee.

\section{Setup and procedures}

A dual-belt force plate-instrumented $(1000 \mathrm{~Hz})$ treadmill with a virtual environment that provided optic flow during walking (Computer Assisted Rehabilitation Environment Extended, CAREN; Motekforce Link, Amsterdam, The Netherlands) and a 12-camera motion capture system $(100 \mathrm{~Hz}$; Vicon Motion Systems, Oxford, UK) were used in this study. Three high definition video cameras recorded video footage of the trials. Five retroreflective markers were attached to anatomical landmarks $(\mathrm{C} 7$, left and right trochanter and left and right hallux), and the three-dimensional coordinates of these markers were tracked by the motion capture system. Participants were secured in a safety harness system throughout the measurements.

Participants first completed 60 -second walking familiarisation trials at speeds of $0.4 \mathrm{~m} / \mathrm{s}$ up to $1.8 \mathrm{~m} / \mathrm{s}$ in $0.2 \mathrm{~m} / \mathrm{s}$ increments and were given sufficient rest (approximately two minutes) before continuing with the recorded trials, comprised of two-to-three-minutelong trials (to ensure a sufficient minimum number of strides) at the same speeds. While participants rested, the stability-normalised walking speed was calculated by fitting a second-order polynomial function to the mean anteroposterior MoS (see below) of the final 10 steps of each walking trial $(0.4 \mathrm{~m} / \mathrm{s}$ to $1.8 \mathrm{~m} / \mathrm{s})$ (McCrum et al. 2018; McCrum et al. 2019b). The theoretical background and data on the effectiveness of this approach are described elsewhere (McCrum et al. 2019b). For each participant, the walking speed that would result in MoS of $0.05 \mathrm{~m}$ was calculated from the function. The perturbation trial then began with 3 to 4 minutes of unperturbed walking at the stabilitynormalised walking speed, followed by 10 unannounced unilateral treadmill belt acceleration perturbations, each occurring every $30-90$ seconds. Participants were told that they would complete a walking balance challenge and to try to continue walking as normally as possible. Participants were not aware of the specifics of the protocol (i.e. limbs to be perturbed, type, number, timing, magnitude of the perturbations). The first and tenth accelerations perturbed the right leg, while the second to ninth accelerations perturbed the left leg. The perturbation was a $3 \mathrm{~m} / \mathrm{s}^{2}$ acceleration of the treadmill belt to a maximum speed equal to $180 \%$ of the stability-normalised walking speed. The acceleration began when the hallux marker of the to-be-perturbed limb passed the hallux marker of the opposite foot in the sagittal plane. The belt decelerated at toe-off of the perturbed limb.

Data processing and margin of stability calculation

Data processing was conducted in MATLAB (2016a, The MathWorks, Inc., Natick). The three-dimensional coordinates of the markers were filtered using a lowpass, second-order, zero-phase Butterworth filter with a $12 \mathrm{~Hz}$ cut-off frequency. Foot touchdown and toe-off were determined as previously described (McCrum et al. 2018, 2019b). The anteroposterior $\operatorname{MoS}\left(\operatorname{MoS}_{\mathrm{AP}}\right)$ at foot touchdown were calculated as the anteroposterior distance between the anterior boundary of the base of support $(\mathrm{BoS})$ and the extrapolated centre of mass $\left(\mathrm{X}_{\mathrm{CoM}}\right)$ (Hof et al. 2005), adapted for our reduced kinematic model (Süptitz et al. 2013; more details, as well as the equation used for the calculation are available in Online Resource 1, eMethods). The mediolateral MoS $\left(\mathrm{MoS}_{\mathrm{ML}}\right)$ were also calculated in a similar manner (mediolateral components instead of anteroposterior), with the exceptions that the treadmill belt velocity was not included in the estimation of CoM velocity and that the $\mathrm{MoS}_{\mathrm{ML}}$ was not determined at foot touchdown, but rather the minimum $\mathrm{MoS}_{\mathrm{ML}}$ during the stance phase was determined (Hak et al. 2012). The MoS was calculated for the following steps: baseline for each perturbation was the mean MoS of the eleventh to second last step before each perturbation (Base); the final step before each perturbation (Pre); and the first eight recovery steps following each perturbation (Post1-8). 
Statistics

To investigate the age-related differences in the responses to novel perturbations and the adaptation to repeated perturbations to one leg, two-way repeated measures ANOVAs with group (young, older) and step (Base, Pre, Post1-Post8) as factors were conducted individually for the first, second and ninth perturbations (the first perturbation of the right leg (novel) and the first (novel) and final (adaptation) perturbations of the left "trained" leg; Pert $1_{\mathrm{R}}, \operatorname{Pert}_{\mathrm{L}}$ and Pert $9_{\mathrm{L}}$, respectively). To evaluate any changes in the $\mathrm{MoS}_{\mathrm{AP}}$ during unperturbed walking that would indicate anticipatory adjustments, a repeated measure mixed model with perturbation number $\left(\right.$ Pert $_{R}$, Pert $2_{L}$, Pert $9_{L}$ and Pert $10_{R}$ ) and age group as factors was conducted. To further investigate which MoS components might be responsible for any observed differences in the responses to the novel perturbations and the adaptation to repeated perturbations to one leg, the same ANOVAs were conducted for the BoS and $\mathrm{X}_{\mathrm{CoM}}$. Finally, the same ANOVAs were conducted for the $\mathrm{MoS}_{\mathrm{ML}}$, as we suspected that lateral instability may also be increased in the older adults during anteroposterior perturbations (McIlroy and Maki 1996). To determine if interlimb transfer of the reactive adaptations occurred in the older adults, twoway repeated measures ANOVAs with perturbation number $\left(\right.$ Pert $_{R}$ and Pert $\left.10_{R}\right)$ and step (Base, Pre, Post1-Post8) as factors were conducted for $\mathrm{MoS}_{\mathrm{AP}}$, BoS and $\mathrm{X}_{\mathrm{CoM}}$. For all ANOVAs, post hoc Bonferroni tests for multiple comparisons were applied. Sphericity of the data was checked, and when required, outcomes were adjusted using the Geisser-Greenhouse epsilon. Significance was set at $\alpha=0.05$. Analyses were performed using GraphPad Prism version 8.02 for Windows (GraphPad Software Inc., La Jolla, California, USA).

\section{Results}

Similar to our previous work in young adults (McCrum et al. 2018, 2019b), means and SDs of the eleventh to second last step before the first perturbation revealed that most participants ( 25 of 30 young adults and 23 of 28 older adults) were within one SD of the desired 0.05 $\mathrm{m} \mathrm{MoS}_{\mathrm{AP}}$ (Fig. 1). The stability-normalised walking speeds (mean $\pm \mathrm{SD}$, range) were $1.32 \pm 0.07 \mathrm{~m} / \mathrm{s}$,
$1.16-1.51 \mathrm{~m} / \mathrm{s}$ for the young adults and $1.31 \pm 0.13$ $\mathrm{m} / \mathrm{s}, 1.01-1.50 \mathrm{~m} / \mathrm{s}$ for the older adults.

All participants were able to recover from the gait perturbations without harness assistance. However, one older adult stopped walking after recovering from the first perturbation, leading to the treadmill stopping. This participant was therefore excluded from the analyses involving Pert $1_{R}$. Two-way repeated measures ANOVAs for Pert $1_{R}$, Pert $2_{L}$ and Pert $9_{L}$ revealed a significant age group effect on $\mathrm{MoS}_{\mathrm{AP}}$ for Pert $1_{\mathrm{R}}$ only $\left(\right.$ Pert $_{\mathrm{R}}: \mathrm{F}_{(1,55)}=14.11, \mathrm{P}=0.0004, \eta_{\mathrm{p}}{ }^{2}=0.204 ;$ Pert $2_{\mathrm{L}}$ : $\mathrm{F}_{(1,56)}=2.968, \mathrm{P}=0.0904, \eta_{\mathrm{p}}{ }^{2}=0.050 ;$ Pert9 $_{\mathrm{L}}: \mathrm{F}_{(1,56)}=$ $\left.0.2948, P=0.5893, \eta_{p}{ }^{2}=0.005\right)$. Significant Step by Age Group interactions were found for Pert $1_{R}$ and $\operatorname{Pert}_{\mathrm{L}}\left(\operatorname{Pert}_{\mathrm{R}}: \mathrm{F}_{(9,495)}=15.55, \mathrm{P}<0.0001, \eta_{\mathrm{p}}{ }^{2}=\right.$ 0.220 ; Pert $2_{\mathrm{L}}: \mathrm{F}_{(9,504)}=8.310, \mathrm{P}<0.0001, \eta_{\mathrm{p}}{ }^{2}=$ 0.129; $\operatorname{Pert9}_{\mathrm{L}}: \mathrm{F}_{(9,504)}=1.576, \mathrm{P}=0.1192, \eta_{\mathrm{p}}{ }^{2}=$ 0.027). Bonferroni tests for multiple comparisons (Fig. 2) revealed that, on average, older adults had $\mathrm{MoS}_{\mathrm{AP}}$ significantly different to Base for at least three steps more than the young adults during Pert $1_{R}$ and Pert $2_{L}$, but during Pert $9_{\mathrm{L}}$, older adults had $\mathrm{MoS}_{\mathrm{AP}}$ significantly different to Base for only one step more than the young adults (five vs. four steps). The young and older adults improved their recovery performance following repeated perturbations $\left(\right.$ Pert $2_{\mathrm{L}}$ to Pert $\left.9_{\mathrm{L}}\right)$ on average by two and three recovery steps, respectively. Complete Bonferroni results can be found in Online Resource 1 (eTables 1 and 2). Regarding the unperturbed walking $\mathrm{MoS}_{\mathrm{AP}}$, we did find a significant perturbation number effect $\left(\mathrm{F}_{(3,166)}=11.44, \mathrm{P}<0.0001\right)$, and Bonferroni post hoc tests revealed significant differences between Pert $1_{R}$ and Pert $9_{L}, \operatorname{Pert} 2_{L}$ and Pert $9_{L}$ and between Pert $2_{L}$ and Pert $10_{R}$ in the younger adults and between Pert $2_{\mathrm{L}}$ and Pert ${ }_{\mathrm{L}}$ in the older adults, but these differences ranged from $0.2 \mathrm{~cm}$ to $0.8 \mathrm{~cm}$ and were therefore not considered to have a meaningful effect on the main results.

Two-way repeated measures ANOVAs for Pert $1_{R}$, Pert $2_{L}$ and Pert $9_{L}$ revealed significant age group effects on BoS for Pert $1_{R}$, Pert $2_{L}$ and Pert $9_{L}\left(\right.$ Pert $_{R}: F_{(1,55)}=$ 7.862, $\mathrm{P}=0.007, \eta_{\mathrm{p}}{ }^{2}=0.125 ; \operatorname{Pert}_{\mathrm{L}}: \mathrm{F}_{(1,56)}=11.75, \mathrm{P}$ $=0.0011, \eta_{\mathrm{p}}{ }^{2}=0.173 ; \operatorname{Pert9}_{\mathrm{L}}: \mathrm{F}_{(1,56)}=9.078, \mathrm{P}=$ $0.0039, \eta_{\mathrm{p}}{ }^{2}=0.139$; Fig. 3). Significant Step by Age Group interactions were found for Pert $1_{R}$, Pert $2_{L}$ and Pert9 $_{\mathrm{L}}\left(\right.$ Pert $_{\mathrm{R}}: \mathrm{F}_{(9,495)}=3.160, \mathrm{P}=0.001, \eta_{\mathrm{p}}{ }^{2}=0.054$; Pert $2_{\mathrm{L}}: \mathrm{F}_{(9,504)}=7.281, \mathrm{P}<0.0001, \eta_{\mathrm{p}}{ }^{2}=0.115$; Pert9 $_{\mathrm{L}}$ : $\mathrm{F}_{(9,504)}=1.987, \mathrm{P}=0.0389, \eta_{\mathrm{p}}{ }^{2}=0.034$; Fig. 3). Bonferroni tests for multiple comparisons (Fig. 3) 


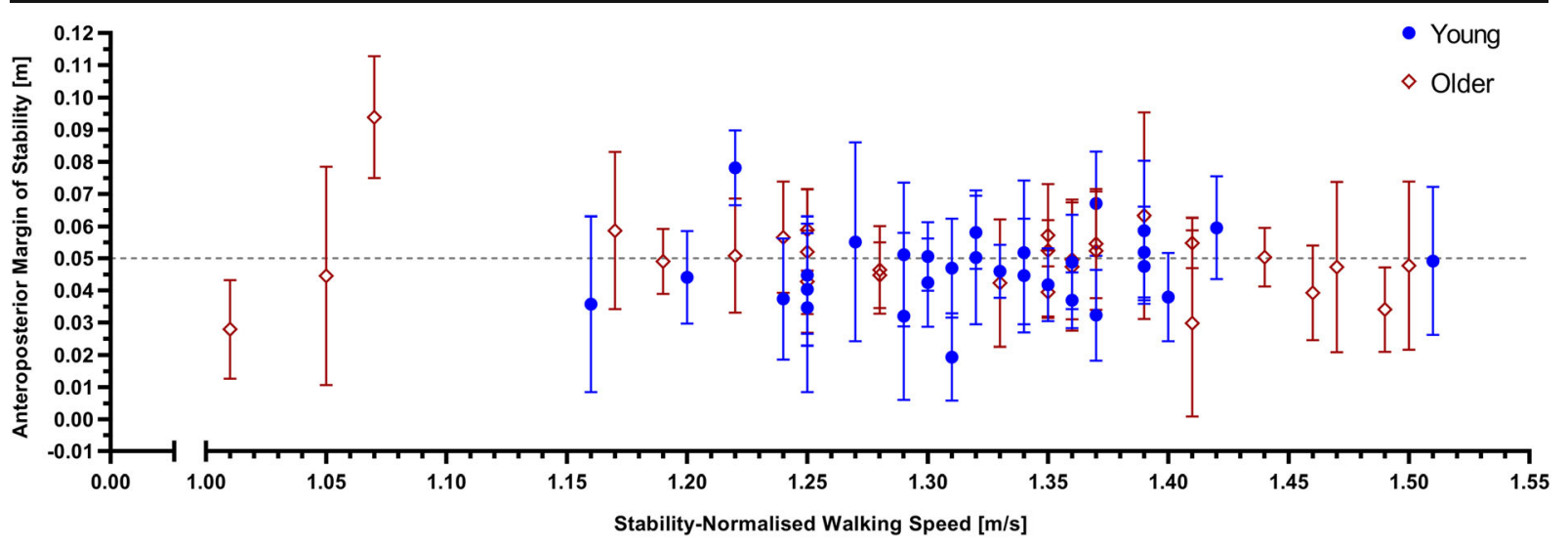

Fig. 1 Anteroposterior margins of stability (means and SDs) of the eleventh to second last step before the first perturbation across the individual stability-normalised walking speeds for young (blue circles) and older (red diamonds) healthy adults

revealed that, on average, older adults had returned to BoS values not significantly different to Base by Post 4 during each of the analysed perturbations. Older adults had a significantly smaller BoS than young adults during Post 2 to Post 4 for Pert 2 , and Post3 and Post4 for Pert9 $_{\mathrm{L}}$. For $\mathrm{X}_{\mathrm{CoM}}$, the ANOVAs revealed significant age group effects $\left(\operatorname{Pert}_{\mathrm{R}}: \mathrm{F}_{(1,55)}=16.26, \mathrm{P}=0.0002, \eta_{\mathrm{p}}{ }^{2}=\right.$ 0.228; Pert $2_{\mathrm{L}}: \mathrm{F}_{(1,56)}=15.64, \mathrm{P}=0.0002, \eta_{\mathrm{p}}{ }^{2}=0.218$; Pert9 ${ }_{\mathrm{L}}: \mathrm{F}_{(1,56)}=9.140, \mathrm{P}=0.0038, \eta_{\mathrm{p}}{ }^{2}=0.140$; Fig. 3) and Step by Age Group interactions $\left(\operatorname{Pert}_{\mathrm{R}}: \mathrm{F}_{(9,495)}=\right.$ $10.45, \mathrm{P}<0.0001, \eta_{\mathrm{p}}{ }^{2}=0.160 ;$ Pert $_{\mathrm{L}}: \mathrm{F}_{(9,504)}=11.84$, $\mathrm{P}<0.0001, \eta_{\mathrm{p}}{ }^{2}=0.175 ; \operatorname{Pert}_{\mathrm{L}}: \mathrm{F}_{(9,504)}=2.440, \mathrm{P}=$ 0.0101, $\eta_{\mathrm{p}}{ }^{2}=0.042$; Fig. 3) for Pert $1_{\mathrm{R}}$, Pert $2_{\mathrm{L}}$ and Pert9 ${ }_{L}$. Bonferroni tests for multiple comparisons revealed that $\mathrm{X}_{\mathrm{CoM}}$ significantly differed between older and young adults from Post 1 to Post 4 for Pert $1_{R}$ and Pert $2_{\mathrm{L}}$ (Fig. 3). Complete Bonferroni results for the BoS and $\mathrm{X}_{\mathrm{CoM}}$ can be found in Online Resource 1 (eTables 3 to 6). Results regarding the $\mathrm{MoS}_{\mathrm{ML}}$ can be found in Online Resource 1 (eResults, eFigure 1, eTables 7 and 8).

Regarding the investigation of interlimb transfer in the older adults $\left(\right.$ Pert $_{\mathrm{R}}$ and Pert $\left.10_{\mathrm{R}}\right)$, no significant perturbation number effects were found for $\mathrm{MoS}_{\mathrm{AP}}$ or $\operatorname{MoS}_{\mathrm{ML}}\left(\mathrm{MoS}_{\mathrm{AP}}: \mathrm{F}_{(1,26)}=2.634, \mathrm{P}=0.1167, \eta_{\mathrm{p}}{ }^{2}=\right.$ $0.092 ; \operatorname{MoS}_{\mathrm{ML}}: \mathrm{F}_{(1,26)}=0.03025, \mathrm{P}=0.8633, \eta_{\mathrm{p}}{ }^{2}=$ 0.001 ; Fig. 4). However, significant perturbation number effects were found for BoS and $\mathrm{X}_{\mathrm{CoM}}\left(\mathrm{BoS}: \mathrm{F}_{(1,26)}=\right.$ 9.104, $\mathrm{P}=0.0056, \eta_{\mathrm{p}}{ }^{2}=0.259 ; \mathrm{X}_{\mathrm{CoM}}: \mathrm{F}_{(1,26)}=18.32, \mathrm{P}$ $=0.0002, \eta_{\mathrm{p}}{ }^{2}=0.413$; Fig. 4), along with significant perturbation number by step interactions for $\mathrm{MoS}_{\mathrm{AP}}$, BoS and $\mathrm{X}_{\mathrm{CoM}}$, but not $\mathrm{MoS}_{\mathrm{ML}}\left(\mathrm{MoS}_{\mathrm{AP}}: \mathrm{F}_{(4.150,107.9)}\right.$ $=16.42, \hat{\varepsilon}=0.4611, \mathrm{P}<0.0001, \eta_{\mathrm{p}}{ }^{2}=0.387$; BoS:
$\mathrm{F}_{(3.029,78.74)}=5.480, \hat{\varepsilon}=0.3365, \mathrm{P}=0.0017, \eta_{\mathrm{p}}{ }^{2}=$ $0.174 ; \mathrm{X}_{\mathrm{CoM}}: \mathrm{F}_{(3.920,101.9)}=12.30, \hat{\varepsilon}=0.4355, \mathrm{P}<$ $0.0001, \eta_{\mathrm{p}}^{2}=0.321 ; \operatorname{MoS}_{\mathrm{ML}}: \mathrm{F}_{(4.056,105.5)}=$ 0.6885 , $\hat{\varepsilon}=0.4507, \mathrm{P}=0.6035, \eta_{\mathrm{p}}{ }^{2}=0.026$; Fig. 4). Bonferroni tests for multiple comparisons are indicated in Fig. 4 and revealed that during Pert $1_{\mathrm{R}}$, the older adults did not return to $\mathrm{MoS}_{\mathrm{AP}}$ values not significantly different to Base during the eight analysed recovery steps, whereas during Pert $10_{R}$, they reached this point by Post6. During Pert $1_{R}$, Post 1 and Post 2 had significantly greater $\mathrm{MoS}_{\mathrm{AP}}$ than during Pert10 $0_{\mathrm{R}}$, but significantly lower $\mathrm{MoS}_{\mathrm{AP}}$ during Post8. BoS was significantly smaller during Pert $1_{R}$ than Pert $10_{R}$ at Post2. This coincided with significant differences between Pert $1_{R}$ and Pert $10_{\mathrm{R}}$ in $\mathrm{X}_{\mathrm{CoM}}$ at Post1, Post2 and Post3, with more anterior $\mathrm{X}_{\mathrm{CoM}}$ during Pert10 $0_{\mathrm{R}}$. No significant differences in $\mathrm{MoS}_{\mathrm{ML}}$ were observed between Pert $1_{R}$ and Pert $10_{\mathrm{R}}$. Complete Bonferroni results for the examination of interlimb transfer can be found in Online Resource 1 (eTables 9 to 16 ).

\section{Discussion}

In the current study, we aimed to determine whether age-related differences in reactive gait stability and its adaptability in response to repeated mechanical gait perturbations are found when the participants' walking speed is normalised to gait stability and whether evidence of interlimb transfer of these adaptations can be observed in healthy older adults. We hypothesised that older adults require more steps to regain stability than younger adults following the initial perturbation to each 


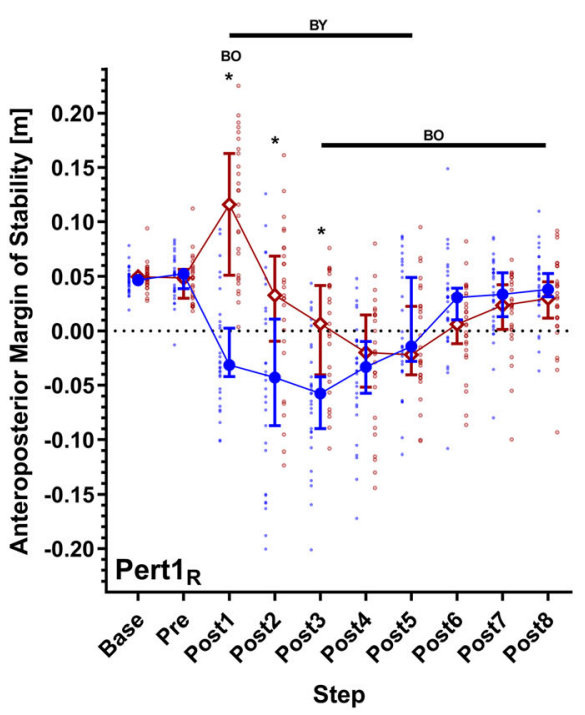

Fig. 2 Median and 95\% confidence intervals (with individual data points) of the anteroposterior margins of stability during the first, second and ninth perturbations $\left(\right.$ Pert $1_{R}$, Pert $2_{L}$, and Pert $9_{L}$, respectively) including unperturbed walking prior to each perturbation (Base), the final step prior to each perturbation (Pre) and the first

leg, despite the stability-normalised walking speed, but that both groups would be able to adapt their gait to improve stability over the repetitions to the left leg. These hypotheses were confirmed, as the older adults required approximately three more steps to regain stability during the first perturbations to each leg than the young adults and after repeated perturbations of the left leg, required approximately three fewer steps to recover than during the first perturbations and were not significantly different to the young adults at any recovery step. These findings confirm previous studies in older adults using slip, trip and surface compliance perturbations (Bhatt et al. 2012; Bierbaum et al. 2010, 2011; Epro et al. 2018a, b; Pai et al. 2014) and extend these to treadmill belt acceleration perturbations during which the walking speed is normalised to stability, ensuring equivalent baseline gait stability and task difficulty. We also hypothesised that evidence of interlimb transfer would be found in the older adults due to them experiencing greater motor error in their early responses. This hypothesis was confirmed, as we found a three-step improvement in the steps to reach $\mathrm{MoS}_{\mathrm{AP}}$ values not significantly different to Base, as well as a more anterior $\mathrm{X}_{\mathrm{CoM}}$ position during Post1 to Post 3 in Pert10 $\mathrm{R}$ compared to Pert $1_{R}$.

Ageing has repeatedly been shown to be associated with poorer performance in regaining stability following unexpected gait perturbations (Bierbaum et al. 2010; Pavol et al. 2002; Süptitz et al. 2013). However, as previously described, potential differences in gait as a result of the walking speed choices in previous studies may have affected these findings (McCrum et al. 2017, 2019b). The current study confirms and consolidates previously reported age-related differences in reactive gait stability, as age differences were observed despite the use of individual stability-normalised walking speeds. We found that these age differences in $\mathrm{MoS}_{\mathrm{AP}}$ were the result of significantly smaller $\mathrm{X}_{\mathrm{CoM}}$ during the first four recovery steps following the first two perturbations and to a lesser extent, smaller BoS during the second to fourth recovery steps following the first and second perturbations. These results indicate that the older adults responded to the treadmill belt acceleration perturbation with a more posterior $\mathrm{X}_{\mathrm{CoM}}$ and smaller BoS than young adults, delaying their stability recovery. This differs to what we have previously observed using a cable-trip setup, where the differences have been observed in the BoS (Epro et al. 2018a; McCrum et al. 2016, 2014), reflecting the differences in perturbation type. By the final perturbation of the trained leg, more posterior $\mathrm{X}_{\mathrm{CoM}}$ (not significant) and smaller $\mathrm{BoS}$ values were still visible in the older adults compared to the young adults, but these no longer led to significantly different $\mathrm{MoS}_{\mathrm{AP}}$ values. Multiple studies have 

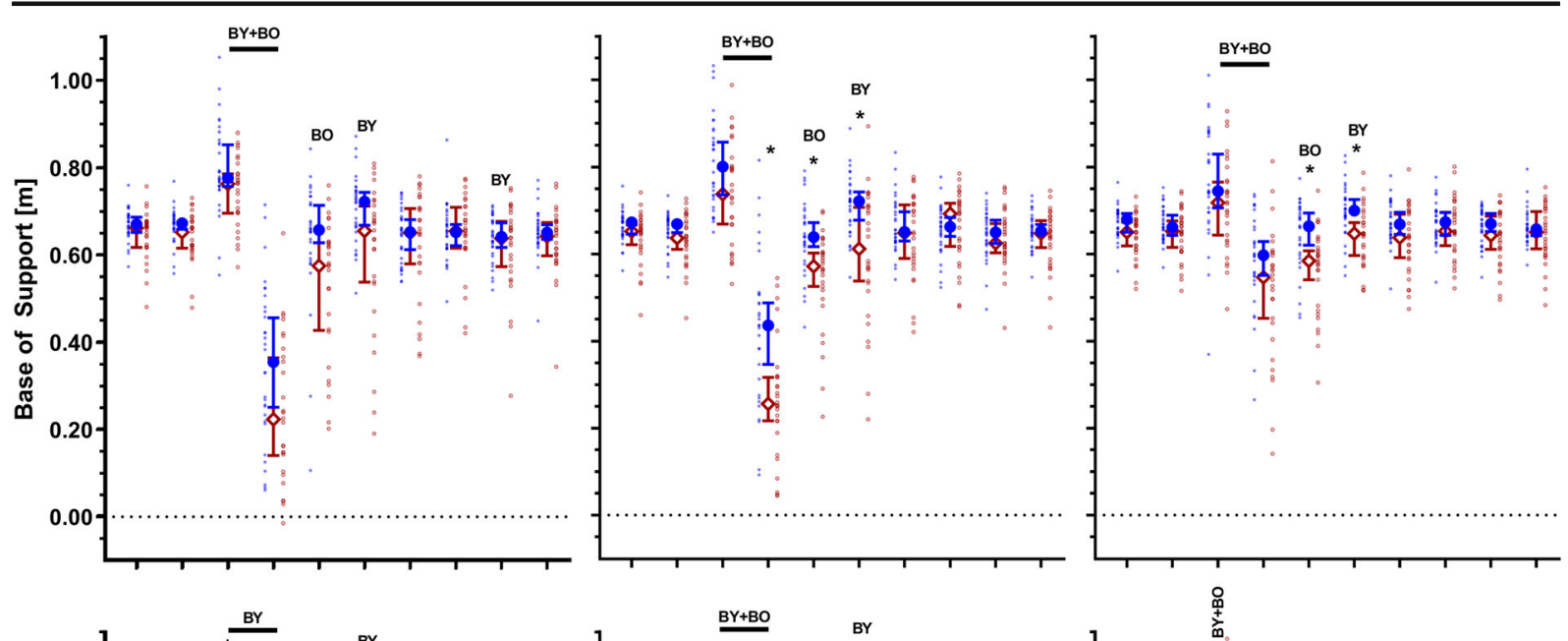

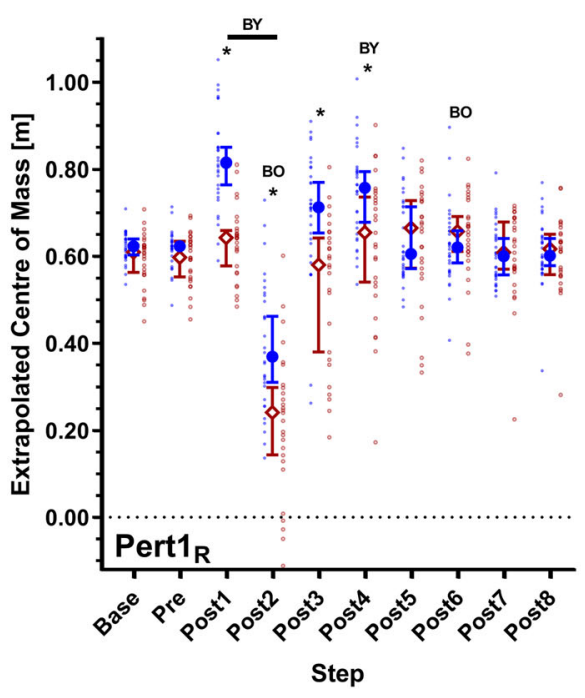

Fig. 3 Median and 95\% confidence intervals (with individual data points) of the anteroposterior base of support and extrapolated centre of mass during the first, second and ninth perturbations $\left(\right.$ Pert $_{\mathrm{R}}$, Pert $_{\mathrm{L}}$ and Pert $9_{\mathrm{L}}$, respectively) including unperturbed walking prior to each perturbation (Base), the final step prior to

demonstrated the ability of healthy adults to reactively adapt gait in response to repeated perturbations (Bhatt et al. 2012; Epro et al. 2018a, b; Pai et al. 2014), and the current study confirms these findings in a treadmill belt acceleration paradigm with stability-normalised walking speeds and walking speed-normalised perturbations. Therefore, we can conclude that potential differences in the initial gait stability or perturbation characteristics likely do not play a large role in whether older adults adapt their response to repeated perturbations.

We previously found little support in young adults for interlimb transfer of reactive gait adaptations following the same protocol as the current study (McCrum et al. 2018). However, we expected that older adults would require more steps to regain stability than younger adults following the initial perturbation to each leg, and thereby they would experience greater motor error in their early responses that may stimulate interlimb transfer. Our results confirmed this expectation, as we found a three-step improvement in the steps to reach $\mathrm{MoS}_{\mathrm{AP}}$ values not significantly different to Base from Pert $1_{R}$ to Pert10 $0_{\mathrm{R}}$. In addition, perturbation number effects on $\mathrm{BoS}$ and $\mathrm{X}_{\mathrm{CoM}}$ were found. The older adults appeared to respond to Pert $1_{\mathrm{R}}$ with a more posterior $\mathrm{X}_{\mathrm{CoM}}$ at Post1 than in Pert $10_{R}$ and with a smaller BoS and posterior $\mathrm{X}_{\mathrm{CoM}}$ at Post2. This alteration in recovery strategy during Post1-3 resulted in the three-step reduction in reaching $\mathrm{MoS}_{\mathrm{AP}}$ values not significantly different to 

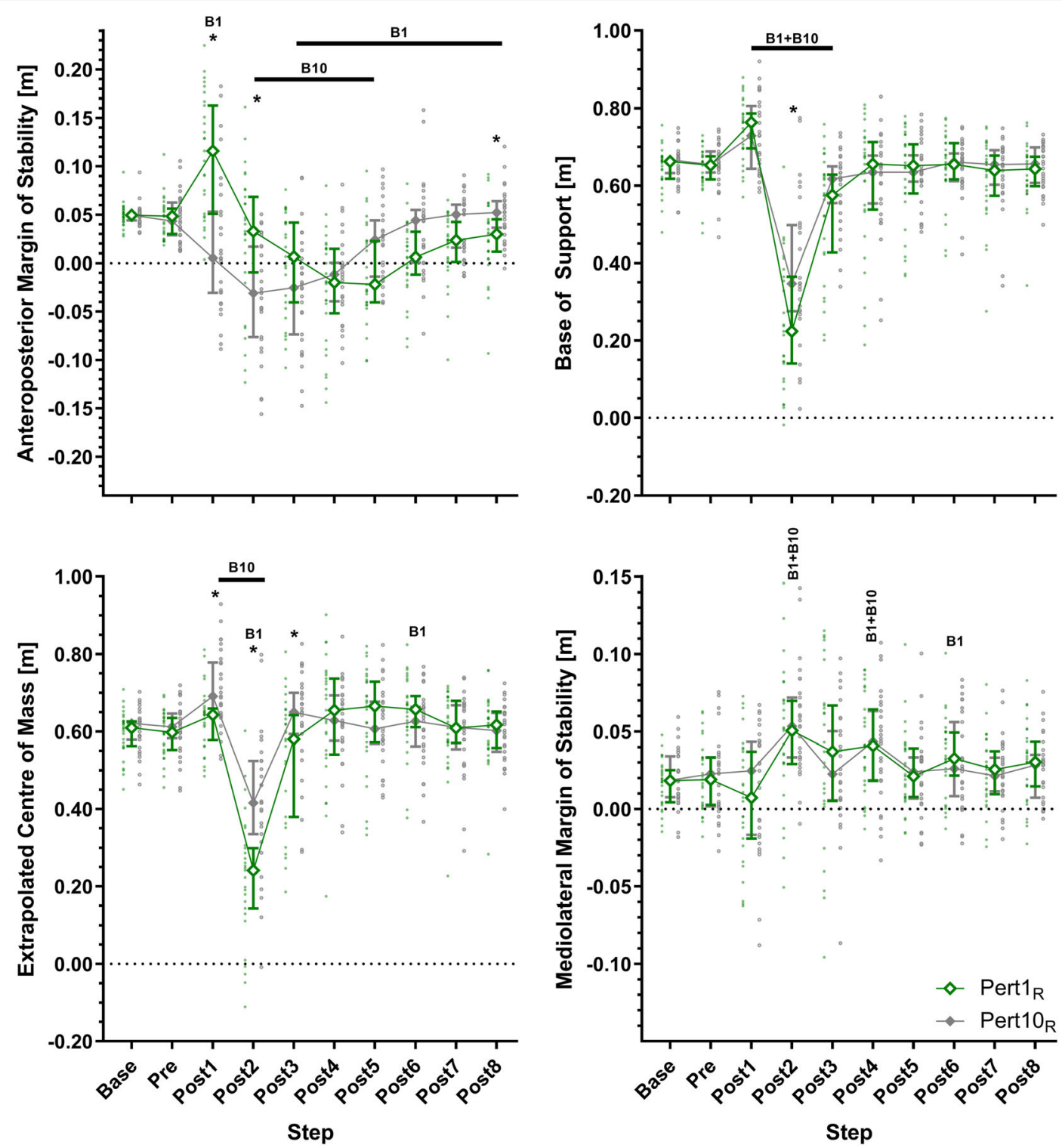

Fig. 4 Median and 95\% confidence intervals (with individual data points) of the anteroposterior margins of stability, base of support, extrapolated centre of mass and mediolateral margins of stability during the first and tenth perturbations (the first and final perturbations to the right leg; Pert $1_{\mathrm{R}}$ and Pert $10_{\mathrm{R}}$, respectively) including unperturbed walking prior to each perturbation (Base), the final

Base. Therefore, it appears that both the overall recovery performance and the altered movement strategy were transferred to the untrained leg. However, as it is well established that learning following such perturbations can occur within a single trial (Liu et al. 2017; Marigold and Patla 2002), we conducted an additional post hoc analysis to determine the extent of trial-to-trial learning for the first two perturbations to the left leg (Pert $2_{\mathrm{L}}$ and

step prior to each perturbation (Pre) and the first eight recovery steps following the perturbations (Post1-8) for older adults. B1 and B10: significant difference to Base for Pert $1_{R}$ and Pert $10_{R}$, respectively $(P<0.05)$. *: significant difference between $P e r t 1_{R}$ and Pert $10_{\mathrm{R}}(\mathrm{P}<0.05)$

Pert $3_{L}$ ), in order to determine if the changes from Pert $1_{R}$ to Pert $10_{\mathrm{R}}$ could be explained by such a phenomenon. Briefly, we did find differences between Pert $2_{\mathrm{L}}$ and Pert $3_{\mathrm{L}}$ but not to the extent that the differences between Pert $1_{\mathrm{R}}$ and Pert $10_{\mathrm{R}}$ could be largely accounted for by the single trial effect. The complete results of this additional analysis can be found in Online Resource 1 (eResults, eFigure 2, eTables 17 and 18). 
An interesting finding of the current study was that the older adults during Pert $1_{\mathrm{R}}$ and Pert $2_{\mathrm{L}}$ demonstrated an increase, rather than a decrease in stability at Post1, whereas the young adults during all perturbations and the older adults during Pert $9_{\mathrm{L}}$ and Pert $10_{\mathrm{R}}$ (Figs. 2 and 4) demonstrated a decrease in stability. This increase was caused by a more posterior $\mathrm{X}_{\mathrm{CoM}}$ during Post1 in Pert $1_{\mathrm{R}}$ and Pert $2_{\mathrm{L}}$, but not a difference in BoS, implying that trunk motion was at least partly responsible. Future work could further investigate this using a kinematic model more suited to assessing trunk motion in detail. We speculate that this may be one potential reason for the observed interlimb transfer of balance recovery performance. While the lower limbs may play very specific roles in perturbation recovery during the first recovery step (i.e. push-off versus swing and placing of the foot), the role of the trunk may be more generalisable across perturbations to different limbs (i.e. counter-rotation to forward balance loss). This may also explain why no clear interlimb transfer occurred in our previous study (McCrum et al. 2018) because young adults appear to have a more anterior $\mathrm{X}_{\mathrm{CoM}}$ position than older adults (Fig. 3). Regarding our analyses of $\mathrm{MoS}_{\mathrm{ML}}$, the results did not reveal any substantial differences with age, and these are discussed in Online Resource 1 (eDiscussion).

A limitation of the current work is that it is unclear if these findings would generalise to populations with reduced locomotor function, and it is these groups that potentially could benefit most from perturbation-based balance training programmes (Gerards et al. 2017). Therefore, interlimb and intertask transfer of adaptations in reactive balance control and the generalisability of these improvements to daily life should be further explored. It could be argued that leg dominance may have affected the results, but due to the bipedal nature of the task, we think this is unlikely. Only one study has specifically investigated the effect of limb dominance on recovery from sudden balance loss and found no differences in performance between stepping with the dominant and nondominant limbs in young and older adults (Madigan and Lloyd 2005). Another limitation worth considering is that despite the evidence provided here that interlimb transfer can occur during a single short perturbation session, this does not necessarily imply that this will be retained over time, as perturbation dose appears to be related to the degree of retention possible (König et al. 2019; Liu et al. 2017).

In conclusion, the current results show that healthy older adults have a decreased ability to cope with unpredictable gait perturbations compared with younger adults, even when their walking speeds are normalised to an equivalent stability value. However, as previous studies have also shown, older healthy adults are capable of reactively adapting their gait to improve their stability following repeated gait perturbations and can then perform similarly to young adults. The current study provides evidence that older adults can transfer improvements in the number of steps required for balance recovery following repeated perturbations to one limb to their recovery following a perturbation to the untrained limb, which in this study was mostly due to an alteration in the $\mathrm{X}_{\mathrm{CoM}}$ position, rather than in the BoS.

Acknowledgements This work was supported by NUTRIM, Maastricht University Medical Centre+ (NUTRIM Graduate Programme grant to C.M.); and CRISP, Maastricht University Medical Centre+ (Kootstra Talent Fellowship to C.M.). The authors thank Paul Willems, Wouter Bijnens and Marissa Gerards for their support during this project. A pre-print version of this work is available at bioRxiv (McCrum et al. 2019a).

Open Access This article is distributed under the terms of the Creative Commons Attribution 4.0 International License (http:// creativecommons.org/licenses/by/4.0/), which permits unrestricted use, distribution, and reproduction in any medium, provided you give appropriate credit to the original author(s) and the source, provide a link to the Creative Commons license, and indicate if changes were made.

\section{References}

Berg WP, Alessio HM, Mills EM, Tong C (1997) Circumstances and consequences of falls in independent communitydwelling older adults. Age Ageing 26:261-268. https://doi. org/10.1093/ageing/26.4.261

Bhatt T, Pai YC (2008) Immediate and latent interlimb transfer of gait stability adaptation following repeated exposure to slips. J Mot Behav 40:380-390. https://doi.org/10.3200 /JMBR.40.5.380-390

Bhatt T, Wening JD, Pai YC (2005) Influence of gait speed on stability: recovery from anterior slips and compensatory stepping. Gait Posture 21:146-156. https://doi.org/10.1016 /j.gaitpost.2004.01.008

Bhatt T, Yang F, Pai YC (2012) Learning to resist gait-slip falls: long-term retention in community-dwelling older adults. Arch Phys Med Rehabil 93:557-564. https://doi. org/10.1016/j.apmr.2011.10.027

Bierbaum S, Peper A, Karamanidis K, Arampatzis A (2010) Adaptational responses in dynamic stability during disturbed walking in the elderly. J Biomech 43:2362-2368. https://doi. org/10.1016/j.jbiomech.2010.04.025

Bierbaum S, Peper A, Karamanidis K, Arampatzis A (2011) Adaptive feedback potential in dynamic stability during 
disturbed walking in the elderly. J Biomech 44:1921-1926. https://doi.org/10.1016/j.jbiomech.2011.04.027

Bieryla KA, Madigan ML, Nussbaum MA (2007) Practicing recovery from a simulated trip improves recovery kinematics after an actual trip. Gait Posture 26:208-213. https://doi. org/10.1016/j.gaitpost.2006.09.010

Emken JL, Reinkensmeyer DJ (2005) Robot-enhanced motor learning: accelerating internal model formation during locomotion by transient dynamic amplification. IEEE Trans Neural Syst Rehabil Eng 13:33-39. https://doi.org/10.1109 /TNSRE.2004.843173

Epro G, McCrum C, Mierau A, Leyendecker M, Brüggemann GP, Karamanidis K (2018a) Effects of triceps surae muscle strength and tendon stiffness on the reactive dynamic stability and adaptability of older female adults during perturbed walking. J Appl Physiol 124:1541-1549. https://doi. org/10.1152/japplphysiol.00545.2017

Epro G, Mierau A, McCrum C, Leyendecker M, Brüggemann GP, Karamanidis K (2018b) Retention of gait stability improvements over 1.5 years in older adults: effects of perturbation exposure and triceps surae neuromuscular exercise. J Neurophysiol 119:2229-2240. https://doi.org/10.1152 /jn.00513.2017

Espy DD, Yang F, Bhatt T, Pai YC (2010) Independent influence of gait speed and step length on stability and fall risk. Gait Posture 32:378-382. https://doi.org/10.1016/j. gaitpost.2010.06.013

Gerards MHG, McCrum C, Mansfield A, Meijer K (2017) Perturbation-based balance training for falls reduction among older adults: Current evidence and implications for clinical practice. Geriatr Gerontol Int 17:2294-2303. https://doi. org/10.1111/ggi.13082

Hak L, Houdijk H, Steenbrink F, Mert A, van der Wurff P, Beek PJ, van Dieen JH (2012) Speeding up or slowing down?: Gait adaptations to preserve gait stability in response to balance perturbations. Gait Posture 36:260-264. https://doi. org/10.1016/j.gaitpost.2012.03.005

Hof AL, Gazendam MG, Sinke WE (2005) The condition for dynamic stability. J Biomech 38:1-8. https://doi. org/10.1016/j.jbiomech.2004.03.025

Kao PC, Srivastava S, Agrawal SK, Scholz JP (2013) Effect of robotic performance-based error-augmentation versus errorreduction training on the gait of healthy individuals. Gait Posture 37:113-120. https://doi.org/10.1016/j. gaitpost.2012.06.025

König M, Epro G, Seeley J, Catala-Lehnen P, Potthast W, Karamanidis K (2019) Retention of improvement in gait stability over 14 weeks due to trip-perturbation training is dependent on perturbation dose. J Biomech 84:243-246. https://doi.org/10.1016/j.jbiomech.2018.12.011

Leech KA, Roemmich RT, Bastian AJ (2018) Creating flexible motor memories in human walking. Sci Rep 8:94. https://doi. org/10.1038/s41598-017-18538-w

Liu X, Bhatt T, Pai YC (2016) Intensity and generalization of treadmill slip training: high or low, progressive increase or decrease? J Biomech 49:135-140. https://doi.org/10.1016/j. jbiomech.2015.06.004

Liu X, Bhatt T, Wang S, Yang F, Pai YC (2017) Retention of the "first-trial effect" in gait-slip among community-living older adults. Geroscience 39:93-102. https://doi.org/10.1007 /s11357-017-9963-0
Madigan ML, Lloyd EM (2005) Age and stepping limb performance differences during a single-step recovery from a forward fall. J Gerontol A Biol Sci Med Sci 60:481-485. https://doi.org/10.1093/gerona/60.4.481

Marchal-Crespo L, Schneider J, Jaeger L, Riener R (2014) Learning a locomotor task: with or without errors? J Neuroeng Rehabil 11:25. https://doi.org/10.1186/17430003-11-25

Marchal-Crespo L, Michels L, Jaeger L, Lopez-Oloriz J, Riener R (2017) Effect of error augmentation on brain activation and motor learning of a complex locomotor task front. Neurosci 11:526. https://doi.org/10.3389/fnins.2017.00526

Marigold DS, Patla AE (2002) Strategies for dynamic stability during locomotion on a slippery surface: effects of prior experience and knowledge. J Neurophysiol 88:339-353. https://doi.org/10.1152/jn.00691.2001

McCrum C, Eysel-Gosepath K, Epro G, Meijer K, Savelberg HH, Brüggemann GP, Karamanidis K (2014) Deficient recovery response and adaptive feedback potential in dynamic gait stability in unilateral peripheral vestibular disorder patients. Physiol Rep 2:e12222. https://doi.org/10.14814/phy2.12222

McCrum C, Epro G, Meijer K, Zijlstra W, Brüggemann GP, Karamanidis K (2016) Locomotor stability and adaptation during perturbed walking across the adult female lifespan. $\mathrm{J}$ Biomech 49:1244-1247. https://doi.org/10.1016/j. jbiomech.2016.02.051

McCrum C, Gerards MHG, Karamanidis K, Zijlstra W, Meijer K (2017) A systematic review of gait perturbation paradigms for improving reactive stepping responses and falls risk among healthy older adults. Eur Rev Aging Phys Act 14:3. https://doi.org/10.1186/s11556-017-0173-7

McCrum C, Karamanidis K, Willems P, Zijlstra W, Meijer K (2018) Retention, savings and interlimb transfer of reactive gait adaptations in humans following unexpected perturbations. Commun Biol 1:230. https://doi.org/10.1038/s42003018-0238-9

McCrum C, Karamanidis K, Grevendonk L, Zijlstra W, Meijer K (2019a) Older adults demonstrate interlimb transfer of reactive gait adaptations to repeated unpredictable gait perturbations. bioRxiv 673574. https://doi.org/10.1101/673574

McCrum C, Willems P, Karamanidis K, Meijer K (2019b) Stability-normalised walking speed: a new approach for human gait perturbation research. J Biomech 87:48-53. https://doi.org/10.1016/j.jbiomech.2019.02.016

McIlroy WE, Maki BE (1996) Age-related changes in compensatory stepping in response to unpredictable perturbations. $\mathrm{J}$ Gerontol A Biol Sci Med Sci 51:M289-M296. https://doi. org/10.1093/gerona/51a.6.m289

Pai YC, Yang F, Bhatt T, Wang E (2014) Learning from laboratory-induced falling: long-term motor retention among older adults. Age 36:9640. https://doi.org/10.1007/s11357014-9640-5

Parijat P, Lockhart TE (2012) Effects of moveable platform training in preventing slip-induced falls in older adults. Ann Biomed Eng 40:1111-1121. https://doi.org/10.1007/s10439011-0477-0

Pavol MJ, Owings TM, Foley KT, Grabiner MD (1999) Gait characteristics as risk factors for falling from trips induced in older adults. J Gerontol A Biol Sci Med Sci 54:M583M590. https://doi.org/10.1093/gerona/54.11.m583 
Pavol MJ, Runtz EF, Edwards BJ, Pai YC (2002) Age influences the outcome of a slipping perturbation during initial but not repeated exposures. J Gerontol A Biol Sci Med Sci 57: M496-M503. https://doi.org/10.1093/gerona/57.8.m496

Roemmich RT, Bastian AJ (2015) Two ways to save a newly learned motor pattern. J Neurophysiol 113:3519-3530. https://doi.org/10.1152/jn.00965.2014

Süptitz F, Moreno Catala M, Brüggemann GP, Karamanidis K (2013) Dynamic stability control during perturbed walking can be assessed by a reduced kinematic model across the adult female lifespan. Hum Mov Sci 32:1404-1414. https://doi.org/10.1016/j.humov.2013.07.008

Talbot LA, Musiol RJ, Witham EK, Metter EJ (2005) Falls in young, middle-aged and older community dwelling adults: perceived cause, environmental factors and injury. BMC Public Health 5:86. https://doi.org/10.1186/1471-2458-5-86
Torres-Oviedo G, Bastian AJ (2012) Natural error patterns enable transfer of motor learning to novel contexts. J Neurophysiol 107:346-356. https://doi.org/10.1152/jn.00570.2011

van Asseldonk EH, Koopman B, van der Kooij H (2011) Locomotor adaptation and retention to gradual and sudden dynamic perturbations. IEEE Int Conf Rehabil Robot 2011: 5975379. https://doi.org/10.1109/ICORR.2011.5975379

Yang F, Bhatt T, Pai YC (2013) Generalization of treadmill-slip training to prevent a fall following a sudden (novel) slip in over-ground walking. J Biomech 46:63-69. https://doi. org/10.1016/j.jbiomech.2012.10.002

Publisher's note Springer Nature remains neutral with regard to jurisdictional claims in published maps and institutional affiliations. 